\title{
Non-singular spherical harmonic expressions of geomagnetic vector and gradient tensor fields in the local north-oriented reference frame
}

\author{
J. Du ${ }^{1,2,3}$, C. Chen ${ }^{1}$, V. Lesur ${ }^{2}$, and L. Wang ${ }^{1,3}$ \\ ${ }^{1}$ Hubei Subsurface Multi-scale Imaging Key Laboratory, Institute of Geophysics \& Geomatics, \\ China University of Geosciences, Wuhan 430074, China \\ ${ }^{2}$ Helmholtz Centre Potsdam, GFZ German Research Centre for Geosciences, Telegrafenberg 14473, Potsdam, Germany \\ ${ }^{3}$ State Key Laboratory of Geodesy and Earth's Dynamics, Chinese Academy of Sciences, Wuhan 430077, China \\ Correspondence to: J. Du (jinsongdu.cug@gmail.com)
}

Received: 27 October 2014 - Published in Geosci. Model Dev. Discuss.: 05 December 2014

Revised: 23 May 2015 - Accepted: 15 June 2015 - Published: 07 July 2015

\begin{abstract}
General expressions of magnetic vector (MV) and magnetic gradient tensor (MGT) in terms of the first- and second-order derivatives of spherical harmonics at different degrees/orders are relatively complicated and singular at the poles. In this paper, we derived alternative non-singular expressions for the MV, the MGT and also the third-order partial derivatives of the magnetic potential field in the local north-oriented reference frame. Using our newly derived formulae, the magnetic potential, vector and gradient tensor fields and also the third-order partial derivatives of the magnetic potential field at an altitude of $300 \mathrm{~km}$ are calculated based on a global lithospheric magnetic field model GRIMM_L120 (GFZ Reference Internal Magnetic Model, version 0.0) with spherical harmonic degrees 16-90. The corresponding results at the poles are discussed and the validity of the derived formulas is verified using the Laplace equation of the magnetic potential field.
\end{abstract}

\section{Introduction}

Compared to the magnetic vector and scalar measurements, magnetic gradients lead to more robust models of the lithospheric magnetic field. The ongoing Swarm mission of the European Space Agency (ESA) provides measurements not only of the vector and scalar data but also an estimate of their east-west gradients (e.g., Olsen et al., 2004, 2015; FriisChristensen et al., 2006). Kotsiaros and Olsen (2012, 2014) proposed to recover the lithospheric magnetic field through magnetic space gradiometry in the same way that has been done for modeling the gravitational potential field from the satellite gravity gradient tensor measurements by the Gravity field and steady-state Ocean Circulation Explorer (GOCE). Purucker (2005), Purucker et al. (2007), Sabaka et al. (2015) and Kotsiaros et al. (2015) also reported efforts to model the lithospheric magnetic field using magnetic gradient information from the satellite constellation. Their results showed that, by using gradient data, the modeled lithospheric magnetic anomaly field has enhanced shorter wavelength content and a much higher quality compared to models built from vector field data. This is because the gradient data can remove the highly time-dependent contributions of the magnetosphere and ionosphere that are correlated between two side-by-side satellites.

The second-order magnetic gradient tensor consists of spatial derivatives highlighting certain structures of the magnetic field (e.g., Schmidt and Clark, 2000, 2006). It can be used to detect the hidden and small-scale magnetized sources (e.g., Pedersen and Rasmussen, 1990; Harrison and Southam, 1991) and to investigate the orientation of the lineated magnetic anomalies (e.g., Blakely and Simpson, 1986). Quantitative magnetic interpretation methods such as the analytic signal, edge detection, spatial derivatives, Euler deconvolution, and transformations, all set in a Cartesian coordinate system (e.g., Blakely, 1995; Purucker and Whaler, 2007; Taylor et al., 2014) also require calculating the higherorder derivatives of the magnetic anomaly field and need to be extended to regional and global scales to handle the curvature of Earth and other planets. Ravat et al. (2002) and Ravat (2011) utilized the analytic signal method and the total 
gradient to interpret the satellite-altitude magnetic anomaly data. Therefore, both the magnetic field modeling and also the geological interpretations require the calculation for the partial derivatives of the magnetic field, possibly at the poles for specific systems of coordinates. Spherical harmonic analysis, established originally by Gauss (1839), is generally used to model the global magnetic internal fields of Earth and other terrestrial planets (e.g., Maus et al., 2008; Langlais et al., 2010; Thébault et al., 2010; Finlay et al., 2010; Lesur et al., 2013; Sabaka et al., 2013; Olsen et al., 2014). Series of spherical harmonic functions themselves made of Schmidt semi-normalized associated Legendre functions (SSALFs) (e.g., Blakely, 1995; Langel and Hinze, 1998) are fitted by least squares to magnetic measurements, giving the spherical harmonic coefficients (i.e., the Gaussian coefficients) defining the model. Kotsiaros and Olsen $(2012,2014)$ presented the MV (magnetic vector) and the MGT (magnetic gradient tensor) using a spherical harmonic representation and, of course, their expressions are singular as they approach the poles. Even if there are satellite data gaps around the poles, it is advisable to use non-singular spherical harmonic expressions for the MV and the MGT in case airborne or shipborne magnetic data are utilized (e.g., Golynsky et al., 2013; Maus, 2010). A rotation of the coordinate system is always possible to avoid the polar singularity, but this solution is very ineffective for large data sets.

In this paper, following Petrovskaya and Vershkov (2006) and Eshagh $(2008,2009)$ for the gravitational gradient tensor in the local north-oriented, orbital reference and geocentric spherical frames, the non-singular expressions in terms of spherical harmonics for the MV, the MGT and the thirdorder derivatives of the magnetic potential field in the specially defined local-north-oriented reference frame (LNORF) are presented. In the next section, the traditional expressions of the MV and the MGT are first stated, some necessary propositions are then proved and, lastly, new non-singular expressions are derived. In Sect. 3, the new formulae are tested using the global lithospheric magnetic field model GRIMM_L120 (GFZ Reference Internal Magnetic Model, version 0.0) (Lesur et al., 2013) and compared with the results by traditional formulae. Finally, some conclusions are drawn and further applications are also discussed.

\section{Methodology}

In this section, the traditional expressions of MV and MGT are presented and their numerical problems are stated. Then, based on some necessary mathematical derivations, new expressions are given.

\subsection{Traditional expressions}

The scalar potential $V$ of Earth's magnetic field in a sourcefree region can be expanded in the truncated series of spher- ical harmonics at the point $P(r, \theta, \varphi)$ with the geocentric distance $r$, co-latitude $\theta$ and longitude $\varphi$ (e.g., Backus et al., 1996):

$$
\begin{array}{r}
V(r, \theta, \phi)=a \sum_{l=1}^{L} \sum_{m=0}^{l}\left(\frac{a}{r}\right)^{l+1} \\
\left(g_{l}^{m} \cos m \phi+h_{l}^{m} \sin m \phi\right) \widetilde{P}_{l}^{m}(\cos \theta),
\end{array}
$$

where $a=6371.2 \mathrm{~km}$ is the radius of Earth's magnetic reference sphere; $\widetilde{P}_{l}^{m}(\cos \theta)$ (or $\widetilde{P}_{l}^{m}$ for simplification) is the SSALF of degree $l$ and order $m ; L$ is the maximum spherical harmonic degree; and $g_{l}^{m}$ and $h_{l}^{m}$ are the geomagnetic harmonic coefficients describing Earth's internal sources.

If considered in the LNORF $\{x, y, z\}$ (e.g., Olsen et al., 2010), where the $z$ axis points downward in the geocentric radial direction, the $x$ axis points to the north, and the $y$ axis towards the east (that is, a right-handed system). At the poles, we define that the $x$ axis points to the meridian of $180^{\circ} \mathrm{E}$ (or $180^{\circ} \mathrm{W}$ ) at the North Pole and of $0^{\circ}$ at the South Pole, which will be discussed in Sect. 3. Therefore, the three components of the MV can be expressed as

$$
\begin{aligned}
& B_{x}(r, \theta, \phi)=-\frac{1}{r} \frac{\partial}{\partial(-\theta)} V(r, \theta, \phi) \\
& =\sum_{l=1}^{L} \sum_{m=0}^{l}\left(\frac{a}{r}\right)^{l+2} \\
& \quad\left(g_{l}^{m} \cos m \phi+h_{l}^{m} \sin m \phi\right)\left[\frac{\mathrm{d}}{\mathrm{d} \theta} \widetilde{P}_{l}^{m}(\cos \theta)\right], \\
& B_{y}(r, \theta, \phi)=-\frac{1}{r \sin \theta} \frac{\partial}{\partial \phi} V(r, \theta, \phi) \\
& =\sum_{l=1}^{L} \sum_{m=0}^{l}\left(\frac{a}{r}\right)^{l+2} m \\
& \quad\left(g_{l}^{m} \sin m \phi-h_{l}^{m} \cos m \phi\right)\left[\frac{1}{\sin \theta} \widetilde{P}_{l}^{m}(\cos \theta)\right], \\
& B_{z}(r, \theta, \phi)=-\frac{\partial}{\partial(-r)} V(r, \theta, \phi) \\
& =-\sum_{l=1}^{L} \sum_{m=0}^{l}(l+1)\left(\frac{a}{r}\right)^{l+2} \\
& \quad\left(g_{l}^{m} \cos m \phi+h_{l}^{m} \sin m \phi\right) \widetilde{P}_{l}^{m}(\cos \theta) .
\end{aligned}
$$

The MGT can be written as (e.g., Kotsiaros and Olsen, 2012)

$\nabla B=\left(\begin{array}{ccc}B_{x x} & B_{x y} & B_{x z} \\ B_{y x} & B_{y y} & B_{y z} \\ B_{z x} & B_{z y} & B_{z z}\end{array}\right)=$

$\left(\begin{array}{lll}\partial B_{x} / \partial x & \partial B_{x} / \partial y & \partial B_{x} / \partial z \\ \partial B_{y} / \partial x & \partial B_{y} / \partial y & \partial B_{y} / \partial z \\ \partial B_{z} / \partial x & \partial B_{z} / \partial y & \partial B_{z} / \partial z\end{array}\right)$,

where nine elements are expressed respectively as

$$
\begin{aligned}
& B_{x x}=\frac{1}{a} \sum_{l=1}^{L} \sum_{m=0}^{l}\left(\frac{a}{r}\right)^{l+3}\left(g_{l}^{m} \cos m \phi+h_{l}^{m} \sin m \phi\right) \\
& \times\left[-\frac{\mathrm{d}^{2}}{\mathrm{~d} \theta^{2}} \widetilde{P}_{l}^{m}(\cos \theta)+(l+1) \widetilde{P}_{l}^{m}(\cos \theta)\right],
\end{aligned}
$$




$$
\begin{aligned}
& B_{x y}=B_{y x}=\frac{1}{a} \sum_{l=1}^{L} \sum_{m=0}^{l}\left(\frac{a}{r}\right)^{l+3} \\
& m\left(g_{l}^{m} \sin m \phi-h_{l}^{m} \cos m \phi\right) \\
& \quad \times\left[-\frac{1}{\sin \theta} \frac{\mathrm{d}}{\mathrm{d} \theta} \widetilde{P}_{l}^{m}(\cos \theta)+\frac{\cos \theta}{\sin ^{2} \theta} \widetilde{P}_{l}^{m}(\cos \theta)\right], \\
& B_{x z}=B_{z x}=\frac{1}{a} \sum_{l=1}^{L} \sum_{m=0}^{l}\left(\frac{a}{r}\right)^{l+3} \\
& (l+2)\left(g_{l}^{m} \cos m \phi+h_{l}^{m} \sin m \phi\right)\left[\frac{\mathrm{d}}{\mathrm{d} \theta} \widetilde{P}_{l}^{m}(\cos \theta)\right], \\
& B_{y y}=\frac{1}{a} \sum_{l=1}^{L} \sum_{m=0}^{l}\left(\frac{a}{r}\right)^{l+3}\left(g_{l}^{m} \cos m \phi+h_{l}^{m} \sin m \phi\right) \\
& \quad \times\left[(l+1) \widetilde{P}_{l}^{m}(\cos \theta)+\frac{m^{2}}{\sin ^{2} \theta} \widetilde{P}_{l}^{m}(\cos \theta)\right. \\
& \left.\quad-\frac{\cos \theta}{\sin \theta} \frac{\mathrm{d}}{\mathrm{d} \theta} \widetilde{P}_{l}^{m}(\cos \theta)\right], \\
& B_{y z}=B_{z y}=\frac{1}{a} \sum_{l=1}^{L} \sum_{m=0}^{l}\left(\frac{a}{r}\right)^{l+3} \\
& (l+2) m\left(g_{l}^{m} \sin m \phi-h_{l}^{m} \cos m \phi\right) \\
& {\left[\frac{1}{\sin \theta} \widetilde{P}_{l}^{m}(\cos \theta)\right]} \\
& B_{z z}=-\frac{1}{a} \sum_{l=1}^{L} \sum_{m=0}^{l}\left(\frac{a}{r}\right)^{l+3}(l+1) \\
& (l+2)\left(g_{l}^{m} \cos m \phi+h_{l}^{m} \sin m \phi\right) \widetilde{P}_{l}^{m}(\cos \theta) .
\end{aligned}
$$

The expressions for $V, B_{z}$ and $B_{z z}$ can be calculated stably even for very high spherical harmonic degrees and orders by using the Holmes and Featherstone (2002a) scheme. However, there exist the singular terms of $1 / \sin \theta$ and $1 / \sin ^{2} \theta$ in Eqs. (2b), (4b), (4d) and (4e) when the computing point approaches to the poles. Moreover, some expressions contain the terms of first- and second-order derivatives of SSALFs, such as Eqs. (2a) and (4a)-(4d). Nevertheless, the up to second-order derivatives for very high degrees and orders of SSALFs can be recursively calculated by the Horner algorithm (Holmes and Featherstone, 2002b). These algorithms are relatively complicated and thus we want to use alternative expressions to avoid the singular terms and also the partial derivatives of SSALFs. It should be stated that our work differs from those presented by Petrovskaya and Vershkov (2006) and Eshagh (2009) in the LNORF and also the associated Legendre functions (ALFs). Nonetheless, the following mathematical derivations are carried out based on their studies on gravity fields.

\subsection{Mathematical derivations}

To deal with the singular terms and first- and second-order derivatives of the SSALFs, some useful mathematical derivations are introduced and proved in the following.

1. Derivation of $\mathrm{d} \widetilde{P}_{l}^{m} / \mathrm{d} \theta$

Based on Eq. (Z.1.44) in Ilk (1983),

$\mathrm{d} P_{l}^{m} / \mathrm{d} \theta=$

$$
0.5\left[(l+m)(l-m+1) P_{l}^{m-1}-P_{l}^{m+1}\right],
$$

and the relation between the ALFs and the SSALFs is

$\widetilde{P}_{l}^{m}=\sqrt{C_{m}(l-m) ! /(l+m) !} P_{l}^{m} ;$

thus, the first-order derivative of the SSALFs can be deduced as

$$
\begin{aligned}
& \mathrm{d} \widetilde{P}_{l}^{m} / \mathrm{d} \theta=a_{l, m} \widetilde{P}_{l}^{m-1}+b_{l, m} \widetilde{P}_{l}^{m+1}, \\
& a_{l, m}=0.5 \sqrt{l+m} \sqrt{l-m+1} \sqrt{C_{m} / C_{m-1}}, \\
& b_{l, m}=-0.5 \sqrt{l+m+1} \sqrt{l-m} \sqrt{C_{m} / C_{m+1}},
\end{aligned}
$$

where

$$
C_{m}=2-\delta_{m, 0}=\left\{\begin{array}{l}
1, m=0 \\
2, m \neq 0
\end{array},\right.
$$

and $\delta$ is the Kronecker delta.

2. Derivation of $\mathrm{d}^{2} \widetilde{P}_{l}^{m} / \mathrm{d} \theta^{2}$

According to Eq. (23) in Eshagh (2008),

$$
\begin{aligned}
& \mathrm{d}^{2} P_{l}^{m} / \mathrm{d} \theta^{2}=0.25(l+m)(l-m+1) \\
& \quad(l+m-1)(l-m+2) P_{l}^{m-2} \\
& \quad-0.25[(l+m)(l-m+1)+(l-m)(l+m+1)] P_{l}^{m} \\
& \quad+0.25 P_{l}^{m+2} .
\end{aligned}
$$

The second-order derivative of the SSALFs can be written as

$$
\begin{aligned}
& \mathrm{d}^{2} \widetilde{P}_{l}^{m} / \mathrm{d} \theta^{2}=c_{l, m} \widetilde{P}_{l}^{m-2}+d_{l, m} \widetilde{P}_{l}^{m} \\
& \quad+e_{l, m} \widetilde{P}_{l}^{m+2}, \\
& c_{l, m}=0.25 \sqrt{l+m} \sqrt{l+m-1} \\
& \sqrt{l-m+2} \sqrt{l-m+1} \sqrt{C_{m} / C_{m-2}}, \\
& d_{l, m}=-0.25[(l+m)(l-m+1)+ \\
& \quad(l-m)(l+m+1)], \\
& e_{l, m}=0.25 \sqrt{l+m+2} \sqrt{l+m+1} \\
& \sqrt{l-m} \sqrt{l-m-1} \sqrt{C_{m} / C_{m+2}} .
\end{aligned}
$$

3. Derivation of $\widetilde{P}_{l}^{m} / \sin \theta$

Using Eq. (Z.1.42) in Ilk (1983),

$$
\begin{aligned}
P_{l}^{m} / \sin \theta & =0.5\left[(l+m)(l+m-1) P_{l-1}^{m-1}\right. \\
& \left.+P_{l-1}^{m+1}\right] / m, m \geq 1,
\end{aligned}
$$

and using Eq. (6) we can obtain that

$$
\begin{aligned}
& \widetilde{P}_{l}^{m} / \sin \theta=f_{l, m} \widetilde{P}_{l-1}^{m-1}+g_{l, m} \widetilde{P}_{l-1}^{m+1}, m \geq 1, \\
& f_{l, m}=0.5 \sqrt{l+m} \sqrt{l+m-1} \\
& \sqrt{C_{m} / C_{m-1}} / m, m \geq 1, \\
& g_{l, m}=0.5 \sqrt{l-m} \sqrt{l-m-1} \\
& \sqrt{C_{m} / C_{m+1}} / m, m \geq 1 .
\end{aligned}
$$


4. Derivation of $\widetilde{P}_{l}^{m} / \sin ^{2} \theta$

Employing Eq. (31) in Eshagh (2008),

$$
\begin{aligned}
& P_{l}^{m} / \sin ^{2} \theta=\{(l+m)(l+m-1) \\
& \quad(l-m+1)(l-m+2) /(m-1) P_{l}^{m-2} \\
& \quad+[(l+m)(l+m-1) \\
& \quad /(m-1)+(l-m)(l-m-1) /(m+1)] P_{l}^{m}, m \geq 2, \\
& \left.\quad+1 /(m+1) P_{l}^{m+2}\right\} /(4 m),
\end{aligned}
$$

and using Eq. (6) we have

$$
\begin{aligned}
& \widetilde{P}_{l}^{m} / \sin ^{2} \theta=h_{l, m} \widetilde{P}_{l}^{m-2}+k_{l, m} \widetilde{P}_{l}^{m} \\
& +n_{l, m} \widetilde{P}_{l}^{m+2}, m \geq 2, \\
& h_{l, m}=0.25 \sqrt{l+m} \sqrt{l+m-1} \sqrt{l-m+1} \\
& \sqrt{l-m+2} \sqrt{C_{m} / C_{m-2} /[m(m-1)], m \geq 2, \quad(13 \mathrm{~b})} \\
& k_{l, m}=0.25[(l+m)(l+m-1) /(m-1)+ \\
& (l-m)(l-m-1) /(m+1)] / m, m \geq 2, \\
& n_{l, m}=0.25 \sqrt{l-m} \sqrt{l-m-1} \sqrt{l+m+2} \sqrt{l+m+1} \\
& \sqrt{C_{m} / C_{m+2}} /[m(m+1)], m \geq 1 .
\end{aligned}
$$

5. Derivation of $\mathrm{d} \widetilde{P}_{l}^{m} /(\sin \theta \mathrm{d} \theta)$

Using Eq. (36) in Eshagh (2008),

$$
\begin{aligned}
& \mathrm{d} P_{l}^{m} /(\sin \theta \mathrm{d} \theta)=0.25\{(l+m)(l+m-1)(l+m-2) \\
& \quad(l-m+1) /(m-1) P_{l-1}^{m-2} \\
& \quad+[(l+m)(l-m+1) /(m-1)-(l+m+1) \quad m \geq 2, \\
& \quad(l+m) /(m+1)] P_{l-1}^{m} \\
& \left.\quad-1 /(m+1) P_{l-1}^{m+2}\right\},
\end{aligned}
$$

and using Eq. (6) we can derive

$$
\begin{aligned}
& \mathrm{d} \widetilde{P}_{l}^{m} /(\sin \theta \mathrm{d} \theta)=o_{l, m} \widetilde{P}_{l-1}^{m-2}+q_{l, m} \widetilde{P}_{l-1}^{m} \\
& +x_{l, m} \widetilde{P}_{l-1}^{m+2}, m \geq 2, \\
& o_{l, m}=0.25 \sqrt{l+m} \sqrt{l+m-1} \sqrt{l+m-2} \\
& \sqrt{l-m+1} \sqrt{C_{m} / C_{m-2}} /(m-1), m \geq 2, \\
& q_{l, m}=0.25 \sqrt{l-m} \sqrt{l+m}[(l-m+1) /(m-1) \\
& -(l+m+1) /(m+1)], m \geq 2, \\
& x_{l, m}=-0.25 \sqrt{(l+m+1)} \sqrt{l-m} \sqrt{l-m-1} \\
& \sqrt{l-m-2} \sqrt{C_{m} / C_{m+2}} /(m+1) .
\end{aligned}
$$

6. Derivation of $\mathrm{d} \widetilde{P}_{l}^{m} /(\sin \theta \mathrm{d} \theta)-\widetilde{P}_{l}^{m} \cos \theta / \sin ^{2} \theta$

According to Petrovskaya and Vershkov (2006) and Eshagh (2009) we can write

$$
\begin{aligned}
& \mathrm{d} P_{l}^{m} /(\sin \theta \mathrm{d} \theta)-P_{l}^{m} \cos \theta / \sin ^{2} \theta \\
& \quad=0.5\left[(m-1)(l+m)(l-m+1) P_{l}^{m-1} m \geq 1,\right. \\
& \left.\quad / \sin \theta-(m+1) P_{l}^{m+1} / \sin \theta\right] / m,
\end{aligned}
$$

and using Eq. (36) in Eshagh (2008) we can obtain

$$
\begin{aligned}
& P_{l}^{m-1} / \sin \theta=0.5[(l-m+2)(l-m+3) \\
& \left.P_{l+1}^{m-2}+P_{l+1}^{m}\right] /(m-1), m \geq 2, \\
& P_{l}^{m+1} / \sin \theta=0.5[(l-m)(l-m+1) \\
& \left.P_{l+1}^{m}+P_{l+1}^{m+2}\right] /(m+1) .
\end{aligned}
$$

Substituting Eq. (17) into the right-hand side of Eq. (16), and after simplification, we can derive

$$
\begin{aligned}
& \mathrm{d} P_{l}^{m} /(\sin \theta \mathrm{d} \theta)-P_{l}^{m} \cos \theta / \sin ^{2} \theta \\
& \quad=0.25[(l+m)(l-m+1)(l-m+2) \\
& \quad(l-m+3) P_{l+1}^{m-2} \quad m \geq 1 . \\
& \left.\quad+2 m(l-m+1) P_{l+1}^{m}-P_{l+1}^{m+2}\right] / m,
\end{aligned}
$$

And combining Eq. (6) we obtain that

$$
\begin{aligned}
& \mathrm{d} \widetilde{P}_{l}^{m} /(\sin \theta \mathrm{d} \theta)-\widetilde{P}_{l}^{m} \cos \theta / \sin ^{2} \theta \\
& \quad=0.25[\sqrt{l+m} \\
& \quad \sqrt{l-m+1} \sqrt{l-m+2} \sqrt{l-m+3} \\
& \quad \sqrt{C_{m} / C_{m-2}} \widetilde{P}_{l+1}^{m-2} \\
& \quad+2 m \sqrt{l-m+1} \sqrt{l+m+1} \widetilde{P}_{l+1}^{m} \\
& \quad-\sqrt{l+m+1} \sqrt{l+m+2} \\
& \left.\quad \sqrt{l+m+3} \sqrt{l-m} \sqrt{C_{m} / C_{m+2}} \widetilde{P}_{l+1}^{m+2}\right] / m,
\end{aligned}
$$

7. Derivation of $\left[(l+1) \sin ^{2} \theta \widetilde{P}_{l}^{m}+m^{2} \widetilde{P}_{l}^{m}-\right.$ $\left.\sin \theta \cos \theta \mathrm{d} \widetilde{P}_{l}^{m} / \mathrm{d} \theta\right] / \sin ^{2} \theta$

Based on lemma 3 in Eshagh (2009),

$$
\begin{aligned}
& \sin \theta \cos \theta \mathrm{d} P_{l}^{m} / \mathrm{d} \theta=m P_{l}^{m}+(l+1) \sin ^{2} \theta P_{l}^{m} \\
& -\sin \theta P_{l+1}^{m+1}
\end{aligned}
$$

and we can derive

$$
\begin{aligned}
& {\left[(l+1) \sin ^{2} \theta P_{l}^{m}+m^{2} P_{l}^{m}-\sin \theta \cos \theta \mathrm{d} P_{l}^{m} / \mathrm{d} \theta\right] / \sin ^{2} \theta} \\
& \quad=m(m-1) P_{l}^{m} / \sin ^{2} \theta+P_{l+1}^{m+1} / \sin \theta .
\end{aligned}
$$

According to Eq. (10) we can write

$$
\begin{aligned}
& P_{l+1}^{m+1} / \sin \theta=0.5[(l+m+2)(l+m+1) \\
& \left.P_{l}^{m}+P_{l}^{m+2}\right] /(m+1) .
\end{aligned}
$$

Inserting Eqs. (12) and (22) into Eq. (21), and after some simplifications, we obtain that

$$
\begin{aligned}
& {\left[(l+1) \sin ^{2} \theta P_{l}^{m}+m^{2} P_{l}^{m}-\sin \theta \cos \theta \mathrm{d} P_{l}^{m} / \mathrm{d} \theta\right] / \sin ^{2} \theta} \\
& \quad=0.25(l+m)(l+m-1)(l-m+1)(l-m+2) P_{l}^{m-2} \\
& \quad+0.25[(l+m)(l+m-1)+(l-m)(l-m-1)(m-1) /(m+1) \\
& \quad+2(l+m+2)(l+m+1) /(m+1)] P_{l}^{m}+0.25 P_{l}^{m+2} .
\end{aligned}
$$


And combining with Eq. (6) we can derive

$$
\begin{aligned}
& {\left[(l+1) \sin ^{2} \theta \widetilde{P}_{l}^{m}+m^{2} \widetilde{P}_{l}^{m}-\sin \theta \cos \theta \mathrm{d} \widetilde{P}_{l}^{m} / \mathrm{d} \theta\right] / \sin ^{2} \theta} \\
& \quad=0.25 \sqrt{l+m} \sqrt{l+m-1} \\
& \quad \sqrt{l-m+1} \sqrt{l-m+2} \sqrt{C_{m} / C_{m-2}} \widetilde{P}_{l}^{m-2} \\
& \quad+0.25[(l+m)(l+m-1)+(l-m)(l-m-1) \\
& \quad(m-1) /(m+1) \\
& \quad+2(l+m+2)(l+m+1) /(m+1)] \widetilde{P}_{l}^{m} \\
& \quad+0.25 \sqrt{l+m+1} \sqrt{l+m+2} \\
& \quad \sqrt{l-m} \sqrt{l-m-1} \sqrt{C_{m} / C_{m+2}} \widetilde{P}_{l}^{m+2} .
\end{aligned}
$$

\subsection{New expressions}

Inserting the corresponding mathematical derivations in the last section into Eqs. (2) and (4), and after some simplifications, the new expressions for MV and MGT can be written as

$$
\begin{aligned}
& B_{x}=\sum_{l=1}^{L} \sum_{m=0}^{l}\left(\frac{a}{r}\right)^{l+2}\left(g_{l}^{m} \cos m \phi+h_{l}^{m} \sin m \phi\right) \\
& \left(a_{l, m}^{x} \widetilde{P}_{l}^{m-1}+b_{l, m}^{x} \widetilde{P}_{l}^{m+1}\right), \\
& B_{y}=\sum_{l=1}^{L} \sum_{m=0}^{l}\left(\frac{a}{r}\right)^{l+2}\left(g_{l}^{m} \sin m \phi-h_{l}^{m} \cos m \phi\right) \\
& \left(a_{l, m}^{y} \widetilde{P}_{l-1}^{m-1}+b_{l, m}^{y} \widetilde{P}_{l-1}^{m+1}\right), \\
& B_{z}(r, \phi, \lambda)=\sum_{l=1}^{L} \sum_{m=0}^{l}\left(\frac{a}{r}\right)^{l+2}\left(g_{l}^{m} \cos m \lambda+h_{l}^{m} \sin m \lambda\right) \\
& \left(a_{l . m}^{z} \widetilde{P}_{l}^{m}\right), \\
& B_{x x}=\frac{1}{a} \sum_{l=1}^{L} \sum_{m=0}^{l}\left(\frac{a}{r}\right)^{l+3}\left(g_{l}^{m} \cos m \phi+h_{l}^{m} \sin m \phi\right) \\
& \left(a_{l, m}^{x x} \widetilde{P}_{l}^{m-2}+b_{l, m}^{x x} \widetilde{P}_{l}^{m}+c_{l, m}^{x x} \widetilde{P}_{l}^{m+2}\right), \\
& B_{x y}=\frac{1}{a} \sum_{l=1}^{L} \sum_{m=0}^{l}\left(\frac{a}{r}\right)^{l+3}\left(g_{l}^{m} \sin m \phi-h_{l}^{m} \cos m \phi\right) \\
& \left(a_{l, m}^{x y} \widetilde{P}_{l+1}^{m-2}+b_{l, m}^{x y} \widetilde{P}_{l+1}^{m}+c_{l, m}^{x y} \widetilde{P}_{l+1}^{m+2}\right), \\
& B_{x z}=\frac{1}{a} \sum_{l=1}^{L} \sum_{m=0}^{l}\left(\frac{a}{r}\right)^{l+3}\left(g_{l}^{m} \cos m \phi+h_{l}^{m} \sin m \phi\right) \\
& \left(a_{l, m}^{x z} \widetilde{P}_{l}^{m-1}+b_{l, m}^{x z} \widetilde{P}_{l}^{m+1}\right), \\
& B_{y y}=\frac{1}{a} \sum_{l=1}^{L} \sum_{m=0}^{l}\left(\frac{a}{r}\right)^{l+3}\left(g_{l}^{m} \cos m \phi+h_{l}^{m} \sin m \phi\right) \\
& \left(a_{l, m}^{y y} \widetilde{P}_{l}^{m-2}+b_{l, m}^{y y} \widetilde{P}_{l}^{m}+c_{l, m}^{y y} \widetilde{P}_{l}^{m+2}\right),
\end{aligned}
$$

$$
\begin{aligned}
& B_{y z}=\frac{1}{a} \sum_{l=1}^{L} \sum_{m=0}^{l}\left(\frac{a}{r}\right)^{l+3}\left(g_{l}^{m} \sin m \lambda-h_{l}^{m} \cos m \lambda\right) \\
& \left(a_{l, m}^{y z} \widetilde{P}_{l-1}^{m-1}+b_{l, m}^{y z} \widetilde{P}_{l-1}^{m+1}\right), \\
& B_{z z}=\frac{1}{a} \sum_{l=1}^{L} \sum_{m=0}^{l}\left(\frac{a}{r}\right)^{l+3} \\
& \left(g_{l}^{m} \cos m \lambda+h_{l}^{m} \sin m \phi\right) a_{l, m}^{z z} \widetilde{P}_{l}^{m},
\end{aligned}
$$

where the corresponding coefficients of the SSALFs are given as follows:

$\left\{\begin{array}{l}a_{l, m}^{x}=0.5 \sqrt{l+m} \sqrt{l-m+1} \sqrt{C_{m} / C_{m-1}} \\ b_{l, m}^{x}=-0.5 \sqrt{l+m+1} \sqrt{l-m} \sqrt{C_{m} / C_{m+1}}\end{array}\right.$,

$\left\{\begin{array}{l}a_{l, m}^{y}=0.5 \sqrt{l+m} \sqrt{l+m-1} \sqrt{C_{m} / C_{m-1}} \\ b_{l, m}^{y}=0.5 \sqrt{l-m} \sqrt{l-m-1} \sqrt{C_{m} / C_{m+1}},\end{array}\right.$

$a_{l, m}^{z}=-(l+1)$,

$$
\left\{\begin{array}{c}
a_{l, m}^{x x}=-0.25 \sqrt{l+m} \sqrt{l+m-1} \sqrt{l-m+2} \\
\sqrt{l-m+1} \sqrt{C_{m} / C_{m-2}} \\
b_{l, m}^{x x}=0.25[(l+m)(l-m+1) \\
+(l-m)(l+m+1)]+(l+1) \\
c_{l, m}^{x x}=-0.25 \sqrt{l+m+2} \sqrt{l+m+1} \\
\sqrt{l-m} \sqrt{l-m-1} \sqrt{C_{m} / C_{m+2}},
\end{array}\right.
$$$$
\left\{\begin{array}{c}
a_{l, m}^{x y}=-0.25 \sqrt{l+m} \sqrt{l-m+1} \\
\sqrt{l-m+2} \sqrt{l-m+3} \sqrt{C_{m} / C_{m-2}} \\
b_{l, m}^{x y}=-0.5 m \sqrt{l-m+1} \sqrt{l+m+1} \\
c_{l, m}^{x y}=0.25 \sqrt{l+m+1} \sqrt{l+m+2} \\
\sqrt{l+m+3} \sqrt{l-m} \sqrt{C_{m} / C_{m+2}},
\end{array}\right.
$$$$
\left\{\begin{array}{c}
a_{l, m}^{x z}=0.5(l+2) \sqrt{l+m} \\
\sqrt{l-m+1} \sqrt{C_{m} / C_{m-1}}=(l+2) a_{l, m}^{x} \\
b_{l, m}^{x z}=-0.5(l+2) \sqrt{l+m+1} \\
\sqrt{l-m} \sqrt{C_{m} / C_{m+1}}=(l+2) b_{l, m}^{x},
\end{array}\right.
$$$$
\int \begin{aligned}
& a_{l, m}^{y y}=0.25 \sqrt{l+m} \sqrt{l+m-1} \\
& \sqrt{l-m+1} \sqrt{l-m+2} \sqrt{C_{m} / C_{m-2}}
\end{aligned}
$$$$
b_{l, m}^{y y}=0.25[(l+m)(l+m-1)+
$$$$
(l-m)(l-m-1)(m-1) /(m+1)
$$$$
+2(l+m+2)(l+m+1) /(m+1)]
$$$$
\left.c_{l, m}^{y y}=0.25 \sqrt{l+m+1} \sqrt{l+m+2}\right]
$$$$
\sqrt{l-m} \sqrt{l-m-1} \sqrt{C_{m} / C_{m+2}},
$$$$
\left\{\begin{array}{c}
a_{l, m}^{y z}=0.5(l+2) \sqrt{l+m} \\
\sqrt{l+m-1} \sqrt{C_{m} / C_{m-1}}=(l+2) a_{l, m}^{y} \\
b_{l, m}^{y z}=0.5(l+2) \sqrt{l-m} \sqrt{l-m-1} \\
\sqrt{C_{m} / C_{m+1}}=(l+2) b_{l, m}^{y},
\end{array}\right.
$$$$
a_{l, m}^{z z}=-(l+1)(l+2)=(l+2) a_{l, m}^{z} .
$$

Furthermore, some other higher-order partial derivatives and their transformations are usually used to image geologic boundaries in magnetic prospecting, such as the higher-order enhanced analytic signal (e.g., Hsu et al., 1996). Therefore, 

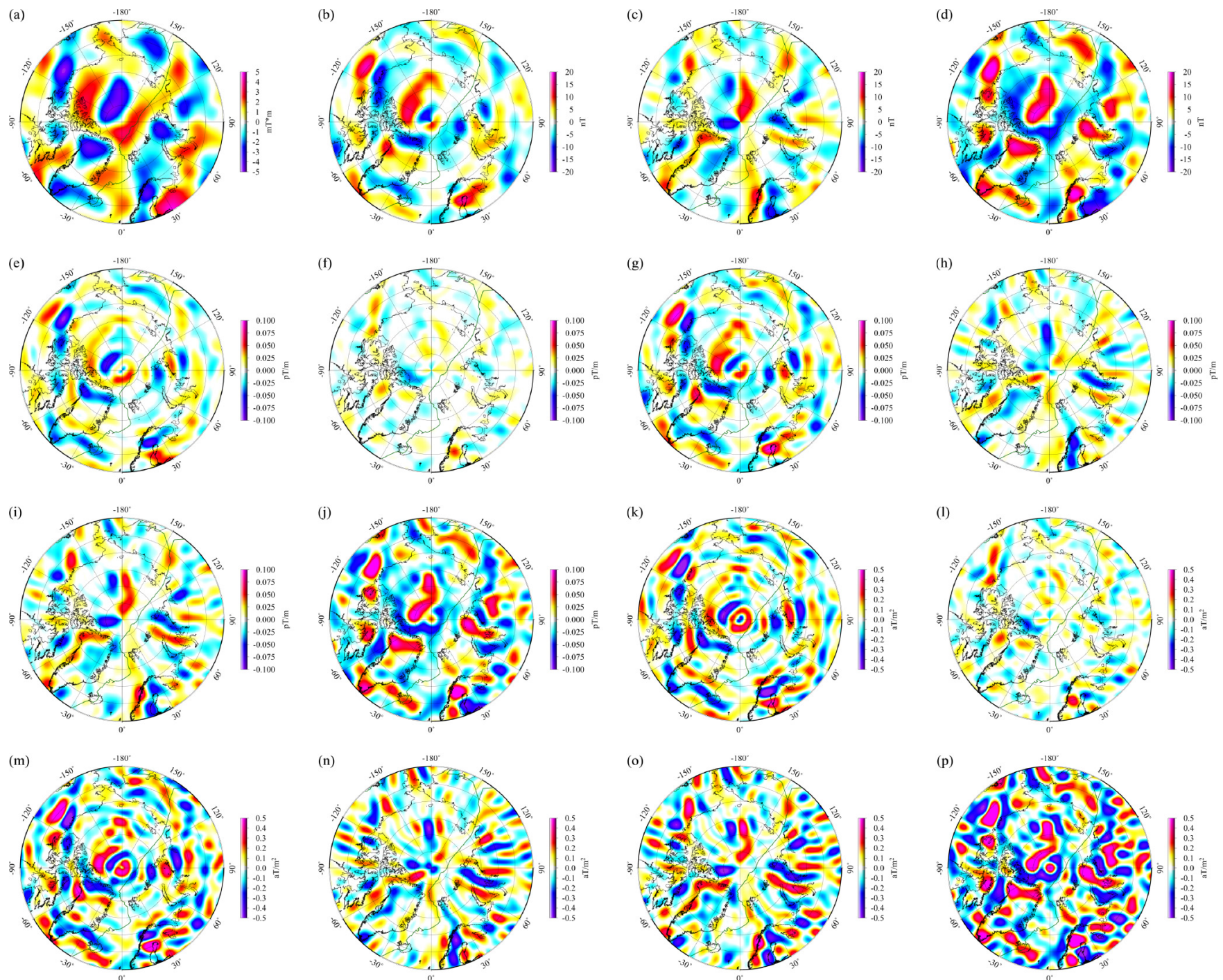

Figure 1. Lithospheric magnetic potential, magnetic vector and its gradient fields and third-order partial derivatives of the magnetic potential field around the North Pole $\left(0^{\circ} \leq \theta \leq 30^{\circ}\right)$ at the altitude of $300 \mathrm{~km}$ as defined by the lithospheric magnetic field model GRIMM_L120 (version 0.0) (Lesur et al., 2013) for spherical harmonic degrees 16-90. (a) is magnetic potential ( $V)$; (b), (c) and (d) are three components $\left(B_{x}, B_{y}\right.$ and $\left.B_{z}\right)$ of the magnetic vector; (e), (f), (g), (h), (i) and (j) are six elements $\left(B_{x x}, B_{x y}, B_{x z}, B_{y y}, B_{y z}\right.$ and $\left.B_{z z}\right)$ of the magnetic gradient tensor; (k), (l), (m), (n), (o) and (p) are six elements $\left(B_{x x z}, B_{x y z}, B_{x z z}, B_{y y z}, B_{y z z}\right.$ and $\left.B_{z z z}\right)$ of third-order partial derivatives of the magnetic potential field, respectively. The dark green lines are the plate boundaries by Bird (2003). All maps are shown in polar stereographic projections.

we also give the third-order partial derivatives of the magnetic potential field as

$$
\begin{aligned}
& B_{x x z}=\frac{\partial B_{x x}}{\partial z}=\frac{\partial^{2} B_{x}}{\partial x \partial z}=\frac{\partial^{2} B_{x}}{\partial z \partial x} \\
& =\frac{1}{a^{2}} \sum_{l=1}^{L} \sum_{m=0}^{l}\left(\frac{a}{r}\right)^{l+4}\left(g_{l}^{m} \cos m \phi+h_{l}^{m} \sin m \phi\right) \\
& \left(a_{l, m}^{x x z} \widetilde{P}_{l}^{m-2}+b_{l, m}^{x x z} \widetilde{P}_{l}^{m}+c_{l, m}^{x x z} \widetilde{P}_{l}^{m+2}\right),
\end{aligned}
$$

$$
\begin{aligned}
& B_{x y z}=\frac{\partial B_{x y}}{\partial z}=\frac{\partial B_{y x}}{\partial z}=\frac{\partial^{2} B_{x}}{\partial y \partial z}=\frac{\partial^{2} B_{x}}{\partial z \partial y}=\frac{\partial^{2} B_{y}}{\partial x \partial z}=\frac{\partial^{2} B_{y}}{\partial z \partial x} \\
& =\frac{1}{a^{2}} \sum_{l=1}^{L} \sum_{m=0}^{l}\left(\frac{a}{r}\right)^{l+4}\left(g_{l}^{m} \sin m \phi-h_{l}^{m} \cos m \phi\right) \\
& \left(a_{l, m}^{x y z} \widetilde{P}_{l+1}^{m-2}+b_{l, m}^{x y z} \widetilde{P}_{l+1}^{m}+c_{l, m}^{x y z} \widetilde{P}_{l+1}^{m+2}\right), \\
& B_{x z z}=\frac{\partial B_{x z}}{\partial z}=\frac{\partial B_{z x}}{\partial z}=\frac{\partial^{2} B_{x}}{\partial z^{2}}=\frac{\partial^{2} B_{z}}{\partial x \partial z}=\frac{\partial^{2} B_{z}}{\partial z \partial x} \\
& =\frac{1}{a^{2}} \sum_{l=1}^{L} \sum_{m=0}^{l}\left(\frac{a}{r}\right)^{l+4}\left(g_{l}^{m} \cos m \phi+h_{l}^{m} \sin m \phi\right) \\
& \left(a_{l, m}^{x z z} \widetilde{P}_{l}^{m-1}+b_{l, m}^{x z z} \widetilde{P}_{l}^{m+1}\right),
\end{aligned}
$$


Table 1. Statistics of the magnetic potential, MV, MGT and third-order partial derivatives of the magnetic potential field around the North Pole $\left(0^{\circ} \leq \theta \leq 30^{\circ}\right)$ at the altitude of $300 \mathrm{~km}$ using the lithospheric magnetic field model GRIMM_L120 (version 0.0) (Lesur et al., 2013) for spherical harmonic degrees 16-90.

\begin{tabular}{lllll}
\hline Magnetic effects & Minimum & Maximum & Mean & Standard deviation \\
\hline$V(\mathrm{mT} \times \mathrm{m})$ & -5.1554771 & +4.7867519 & +0.0828017 & \pm 1.7377648 \\
$B_{x}(\mathrm{nT})$ & -14.7389250 & +17.6917740 & -0.0890689 & \pm 4.9797007 \\
$B_{y}(\mathrm{nT})$ & -15.1297000 & +13.6053000 & +0.0010738 & \pm 4.8239313 \\
$B_{z}(\mathrm{nT})$ & -19.8715270 & +25.3666030 & -0.1988485 & \pm 6.7066701 \\
$B_{x x}\left(\mathrm{pT} \mathrm{m}^{-1}\right)$ & -0.1054684 & +0.0621351 & +0.0001872 & \pm 0.0215871 \\
$B_{x y}\left(\mathrm{pT} \mathrm{m}^{-1}\right)$ & -0.0410371 & +0.0491030 & +0.0000003 & \pm 0.0115018 \\
$B_{x z}\left(\mathrm{pT} \mathrm{m}^{-1}\right)$ & -0.0929498 & +0.1082861 & +0.0006867 & \pm 0.0247522 \\
$B_{y y}\left(\mathrm{pT} \mathrm{m}^{-1}\right)$ & -0.0726248 & +0.0505990 & -0.0004789 & \pm 0.0186580 \\
$B_{y z}\left(\mathrm{pT} \mathrm{m}^{-1}\right)$ & -0.0868184 & +0.0826627 & +0.0000058 & \pm 0.0228174 \\
$B_{z z}\left(\mathrm{pT} \mathrm{m}^{-1}\right)$ & -0.1015986 & +0.1511038 & +0.0002917 & \pm 0.0336965 \\
$B_{x x}+B_{y y}+B_{z z}\left(\mathrm{pT} \mathrm{m}^{-1}\right)$ & $-2.012 \times 10^{-15}$ & $+2.026 \times 10^{-15}$ & $+8.085 \times 10^{-19}$ & $\pm 5.101 \times 10^{-16}$ \\
$B_{x x z}\left(\mathrm{aT} \mathrm{m}^{-2}\right)$ & -0.7589853 & +0.4794999 & +0.0002436 & \pm 0.1537058 \\
$B_{x y z}\left(\mathrm{aT} \mathrm{m}^{-2}\right)$ & -0.2628265 & +0.3734132 & -0.0000004 & \pm 0.0734794 \\
$B_{x z z}\left(\mathrm{aT} \mathrm{m}^{-2}\right)$ & -0.7067652 & +0.8470055 & +0.0140820 & \pm 0.1752880 \\
$B_{y y z}\left(\mathrm{aT} \mathrm{m}^{-2}\right)$ & -0.5259662 & +0.4076568 & -0.0134321 & \pm 0.1370902 \\
$B_{y z z}\left(\mathrm{aT} \mathrm{m}^{-2}\right)$ & -0.6058631 & +0.6396412 & +0.0000341 & \pm 0.1448002 \\
$B_{z z z}\left(\mathrm{aT} \mathrm{m}^{-2}\right)$ & -0.7609268 & +1.1697371 & +0.0131885 & \pm 0.2421663 \\
\hline
\end{tabular}

$$
\begin{aligned}
& B_{y y z}=\frac{\partial B_{y y}}{\partial z}=\frac{\partial^{2} B_{y}}{\partial y \partial z}=\frac{\partial^{2} B_{y}}{\partial z \partial y} \\
& =\frac{1}{a^{2}} \sum_{l=1}^{L} \sum_{m=0}^{l}\left(\frac{a}{r}\right)^{l+4}\left(g_{l}^{m} \cos m \phi+h_{l}^{m} \sin m \phi\right) \\
& \left(a_{l, m}^{y y z} \widetilde{P}_{l}^{m-2}+b_{l, m}^{y y z} \widetilde{P}_{l}^{m}+c_{l, m}^{y y z} \widetilde{P}_{l}^{m+2}\right), \\
& B_{y z z}=\frac{\partial B_{y z}}{\partial z}=\frac{\partial B_{z y}}{\partial z}=\frac{\partial^{2} B_{y}}{\partial z^{2}}=\frac{\partial^{2} B_{z}}{\partial y \partial z}=\frac{\partial^{2} B_{z}}{\partial z \partial y} \\
& =\frac{1}{a^{2}} \sum_{l=1}^{L} \sum_{m=0}^{l}\left(\frac{a}{r}\right)^{l+4}\left(g_{l}^{m} \sin m \lambda-h_{l}^{m} \cos m \lambda\right) \\
& \left(a_{l, m}^{y z z} \widetilde{P}_{l-1}^{m-1}+b_{l, m}^{y z z} \widetilde{P}_{l-1}^{m+1}\right), \\
& B_{z z z}=\frac{\partial^{2} B_{z}}{\partial z^{2}} \\
& =\frac{1}{a^{2}} \sum_{l=1}^{L} \sum_{m=0}^{l}\left(\frac{a}{r}\right)^{l+4} \\
& \left(g_{l}^{m} \cos m \phi+h_{l}^{m} \sin m \phi\right) a_{l, m}^{z z z} \widetilde{P}_{l}^{m},
\end{aligned}
$$

where the corresponding coefficients of the SSALFs are presented as

$$
\begin{aligned}
& \left\{\begin{array}{l}
a_{l, m}^{x x z}=(l+3) a_{l, m}^{x x} \\
b_{l, m}^{x x z}=(l+3) b_{l, m}^{x x} \\
c_{l, m}^{x x z}=(l+3) c_{l, m}^{x x},
\end{array}\right. \\
& \left\{\begin{array}{l}
a_{l, m}^{x y z}=(l+3) a_{l, m}^{x y} \\
b_{l, m}^{x y z}=(l+3) b_{l, m}^{x y} \\
c_{l, m}^{x y z}=(l+3) c_{l, m}^{x y},
\end{array}\right. \\
& \left\{\begin{array}{l}
a_{l, m}^{x z z}=0.5(l+2)(l+3) \\
\sqrt{l+m} \sqrt{l-m+1} \sqrt{C_{m} / C_{m-1}} \\
=(l+2)(l+3) a_{l, m}^{x}=(l+3) a_{l, m}^{x z} \\
b_{l, m}^{x z z}=-0.5(l+2)(l+3) \\
\sqrt{l+m+1} \sqrt{l-m} \sqrt{C_{m} / C_{m+1}} \\
=(l+2)(l+3) b_{l, m}^{x}=(l+3) b_{l, m}^{x z},
\end{array}\right.
\end{aligned}
$$

$a_{l, m}^{z z z}=-(l+1)(l+2)(l+3)=(l+3) a_{l, m}^{z z}$

$=(l+2)(l+3) a_{l, m}^{z}$.

In this way, we avoid computing recursively the SSALFs with singular terms, their first- and second-order derivatives as in the traditional formulae. The cost is only to calculate two additional degrees and orders for the SSALFs at most. It should be noted that, in this study, we use the conventional form of SSALF that if $m<0$, then $\widetilde{P}_{l}^{m}=(-1)^{|m|} \widetilde{P}_{l}^{|m|}$ and if $m>l$, then $\widetilde{P}_{l}^{m}=0$.

\section{Numerical investigation and discussion}

We test the derived expressions and the numerical implementation in $\mathrm{C} / \mathrm{C}++$ by calculating the magnetic potential, magnetic vector and its gradients and also the third-order partial derivatives of the magnetic potential field on a grid with $0.125^{\circ} \times 0.125^{\circ}$ cell size at the altitude of $300 \mathrm{~km}$ relative to Earth's magnetic reference sphere using the lithospheric magnetic field model GRIMM_L120 (version 0.0) defined 
Table 2. Statistics of the magnetic potential, MV, MGT and third-order partial derivatives of the magnetic potential field around the South Pole $\left(150^{\circ} \leq \theta \leq 180^{\circ}\right)$ at the altitude of $300 \mathrm{~km}$ using the lithospheric magnetic field model GRIMM_L120 (version 0.0) (Lesur et al., 2013) for spherical harmonic degrees 16-90.

\begin{tabular}{lllll}
\hline Magnetic effects & Minimum & Maximum & Mean & Standard deviation \\
\hline$V(\mathrm{mT} \times \mathrm{m})$ & -3.3267455 & +4.6543369 & +0.0801853 & \pm 1.2427083 \\
$B_{x}(\mathrm{nT})$ & -11.440070 & +15.9109730 & +0.3451248 & \pm 3.5403285 \\
$B_{y}(\mathrm{nT})$ & -9.1169009 & +15.0436160 & -0.0001605 & \pm 3.1560093 \\
$B_{z}(\mathrm{nT})$ & -22.202857 & +14.5020010 & -0.3022955 & \pm 4.7971494 \\
$B_{x x}\left(\mathrm{pT} \mathrm{m}^{-1}\right)$ & -0.0579914 & +0.0704617 & +0.0000845 & \pm 0.0166266 \\
$B_{x y}\left(\mathrm{pT} \mathrm{m}^{-1}\right)$ & -0.0364002 & +0.0308075 & -0.0000006 & \pm 0.0074702 \\
$B_{x z}\left(\mathrm{pT} \mathrm{m}^{-1}\right)$ & -0.0741850 & +0.0831062 & +0.0019925 & \pm 0.0187492 \\
$B_{y y}\left(\mathrm{pT} \mathrm{m}^{-1}\right)$ & -0.0569493 & +0.0706456 & +0.0019055 & \pm 0.0143289 \\
$B_{y z}\left(\mathrm{pT} \mathrm{m}^{-1}\right)$ & -0.0599346 & +0.0897167 & -0.0000012 & \pm 0.0154623 \\
$B_{z z}\left(\mathrm{pT} \mathrm{m}^{-1}\right)$ & -0.1367168 & +0.0735795 & -0.0019900 & \pm 0.0258066 \\
$B_{x x}+B_{y y}+B_{z z}\left(\mathrm{pT} \mathrm{m}^{-1}\right)$ & $-1.027 \times 10^{-15}$ & $+2.012 \times 10^{-15}$ & $+1.113 \times 10^{-18}$ & $\pm 5.059 \times 10^{-16}$ \\
$B_{x x z}\left(\mathrm{aT} \mathrm{m}^{-2}\right)$ & -0.4605216 & +0.5307263 & +0.0011232 & \pm 0.1328515 \\
$B_{x y z}\left(\mathrm{aT} \mathrm{m}^{-2}\right)$ & -0.2840344 & +0.2947601 & -0.0000015 & \pm 0.0526629 \\
$B_{x z z}\left(\mathrm{aT} \mathrm{m}^{-2}\right)$ & -0.5686811 & +0.5634376 & 0.0181792 & \pm 0.1497829 \\
$B_{y y z}\left(\mathrm{aT} \mathrm{m}^{-2}\right)$ & -0.4262850 & +0.5819095 & +0.0186968 & \pm 0.1169641 \\
$B_{y z z}\left(\mathrm{aT} \mathrm{m}^{-2}\right)$ & -0.6194116 & +0.6520948 & -0.0000118 & \pm 0.1085051 \\
$B_{z z z}\left(\mathrm{aT} \mathrm{m}^{-2}\right)$ & -1.0199774 & +0.5863084 & -0.0198200 & \pm 0.2084566 \\
\hline
\end{tabular}

by Lesur et al. (2013). The magnetic potential, MV, MGT and the third-order partial derivatives of the magnetic potential field in the two polar regions mapped by the lithospheric field model with spherical harmonic degrees 16-90 are shown in Fig. 1 and Fig. 2, respectively. The corresponding statistics around the North Pole and South Pole are, respectively, presented in Tables 1 and 2. A simple test is that the MGT meets Laplace's equation of the potential field; that is, the trace of the MGT should be equal to zero. Our numerical results show that the amplitudes of $B_{x x}+B_{y y}+B_{z z}$ in the North Pole and South Pole regions are in the range of $\left[-2.012 \times 10^{-15} \mathrm{pT} \mathrm{m}^{-1}\right.$ to $\left.+2.026 \times 10^{-15} \mathrm{pT} \mathrm{m}^{-1}\right]$ $\left(1\right.$ Tesla $\left.=10^{3} \mathrm{mT}=10^{9} \mathrm{nT}=10^{12} \mathrm{pT}=10^{18} \mathrm{aT}\right)$, respectively. The relative error is almost equal to the machine's accuracy. Therefore, this feature proves the validity of our derived formulae. In addition, as shown in Figs. 1 and 2, it is obvious that the MGT and also the third-order partial derivatives of the magnetic potential field enhance the lineation and contacts at the satellite altitude. It also reveals some small-scale anomalies, which is very helpful for further geological interpretation. A core field model with spherical harmonic degrees/orders 1-15 is also used for testing and the results, not shown here, indicate the correctness of the formulae in the full range of the spherical harmonic degrees/orders, where the computational stability of the Legendre function with ultrahigh-order is not considered.

Furthermore, the computed magnetic fields are smooth near the poles and do not have the singularities, but some components have the dependence on the direction of the reference frame at the poles. As shown in Fig. 3, the magnetic potential $V$, the $B_{z}, B_{z z}$ and $B_{z z z}$ components at the poles are independent of the direction of the $x_{\mathrm{P}}$ and $y_{\mathrm{P}}$ axes; while changing with the direction of the $x_{\mathrm{P}}$ and $y_{\mathrm{P}}$ axes at the poles, the $B_{x}, B_{y}, B_{x z}, B_{y z}, B_{x z z}$ and $B_{y z z}$ components have a period of $360^{\circ}$ and the $B_{x x}, B_{x y}, B_{y y}, B_{x x z}, B_{x y z}$ and $B_{y y z}$ components have a period of $180^{\circ}$. These variations can be accurately described by a sine or cosine function relating to the horizontal rotation of the reference frame and the differences among these magnetic effects are magnitude, period and initial phase. Therefore, the $B_{x}, B_{y}, B_{x z}, B_{y z}, B_{x x}$, $B_{x y}, B_{y y}, B_{x z z}, B_{y z z} B_{x x z}, B_{x y z}$ and $B_{y y z}$ components are not smooth at/across the poles. Moreover, to determine the single value at the poles (Figs. 1,2) we specially define that the $x$ axis points to the meridian of $180^{\circ} \mathrm{E}$ (or $180^{\circ} \mathrm{W}$ ) at the North Pole and of $0^{\circ}$ at the South Pole, that is, the LNORF moving from Greenwich meridian to the poles.

Compared with the traditional formulae in Sect. 2.1, there are two advantages of our derived formulae in Sect. 2.3. On the one hand, the traditional up to second-order derivatives are removed in the new formulae; therefore, the relatively complicated method by Horner's recursive algorithm (Holmes and Featherstone, 2002b) can be avoided. On the other hand, the singular terms of $1 / \sin \theta$ and $1 / \sin ^{2} \theta$ are removed in the new formulae; consequently, the scale factor of e.g., $10^{-280}$ (Holmes and Featherstone, 2002a, b) is not required when the computing point approaches the poles, and the magnetic fields at the poles can also be calculated in the defined reference frame. In fact, there are differences between the results by our expressions and those by Horner's recursive algorithm; for instance, if using the same model and the parameters as those in Figs. 1 and 2, the differences 

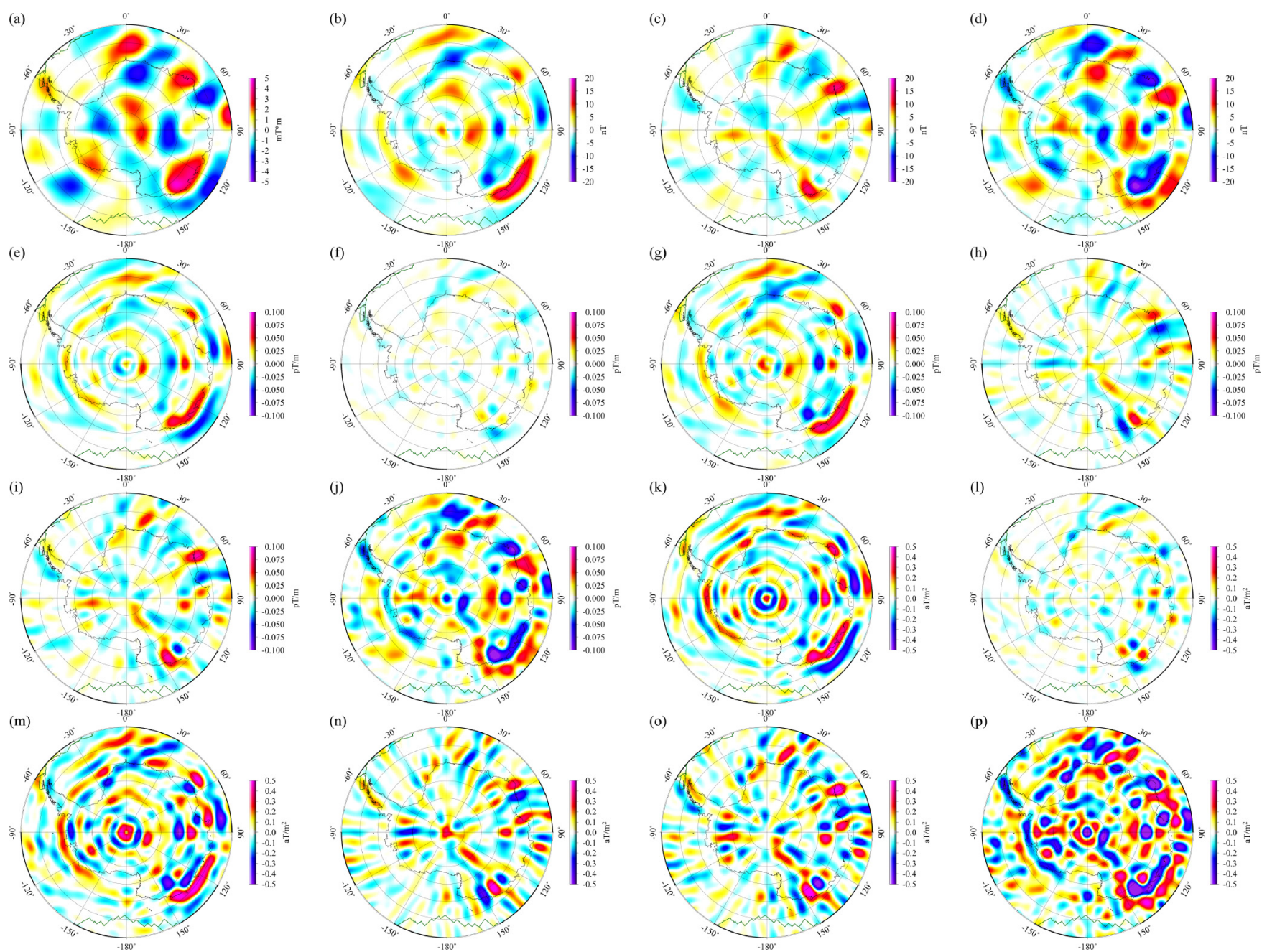

Figure 2. Lithospheric magnetic potential, magnetic vector and its gradient fields and third-order partial derivatives of the magnetic potential field around the South Pole $\left(150^{\circ} \leq \theta \leq 180^{\circ}\right)$ at the altitude of $300 \mathrm{~km}$ as defined by the lithospheric magnetic field model GRIMM_L120 (version 0.0) (Lesur et al., 2013) for spherical harmonic degrees 16-90. (a) is magnetic potential ( $V)$, (b), (c) and (d) are three components $\left(B_{x}, B_{y}\right.$ and $\left.B_{z}\right)$ of the magnetic vector; (e), (f), (g), (h), (i) and (j) are six elements $\left(B_{x x}, B_{x y}, B_{x z}, B_{y y}, B_{y z}\right.$ and $\left.B_{z z}\right)$ of the magnetic gradient tensor; (k), (l), (m), (n), (o) and (p) are six elements $\left(B_{x x z}, B_{x y z}, B_{x z z}, B_{y y z}, B_{y z z}\right.$ and $\left.B_{z z z}\right)$ of third-order partial derivatives of the magnetic potential field, respectively. The dark green lines are the plate boundaries by Bird (2003). All maps are shown in polar stereographic projections.

of the three components $B_{x}, B_{y}$ and $B_{z}$ are at a level of $\left[-3 \times 10^{-11} \mathrm{nT}\right.$ to $\left.+3 \times 10^{-11} \mathrm{nT}\right]$.

\section{Conclusions}

We develop in this paper the new expressions for the MV, the MGT and the third-order partial derivatives of the magnetic potential field in terms of spherical harmonics. The traditional expressions have complicated forms involving firstand second-order derivatives of the SSALFs and are singular when approaching the poles. Our newly derived formulae do not contain the first- and second-order derivatives of the SSALFs and remove the singularities at the poles. However, our formulae are derived in the spherical LNORF with specific definition at the poles. For a future application to the magnetic data of a satellite gradiometry mission (e.g., Kotsiaros and Olsen, 2014), it is necessary to describe the MV and the MGT in the local orbital or other reference frame, where the new MV and MGT are the linear functions of the MV and the MGT in the LNORF with coefficients related to the satellite track azimuth (e.g., Petrovskaya and Vershkov, 2006) or other rotation angles. The other main purpose of this paper is, in the future, to contribute to the signal processing and the geophysical and geological interpretation of the global lithospheric magnetic field model, especially near polar areas. 

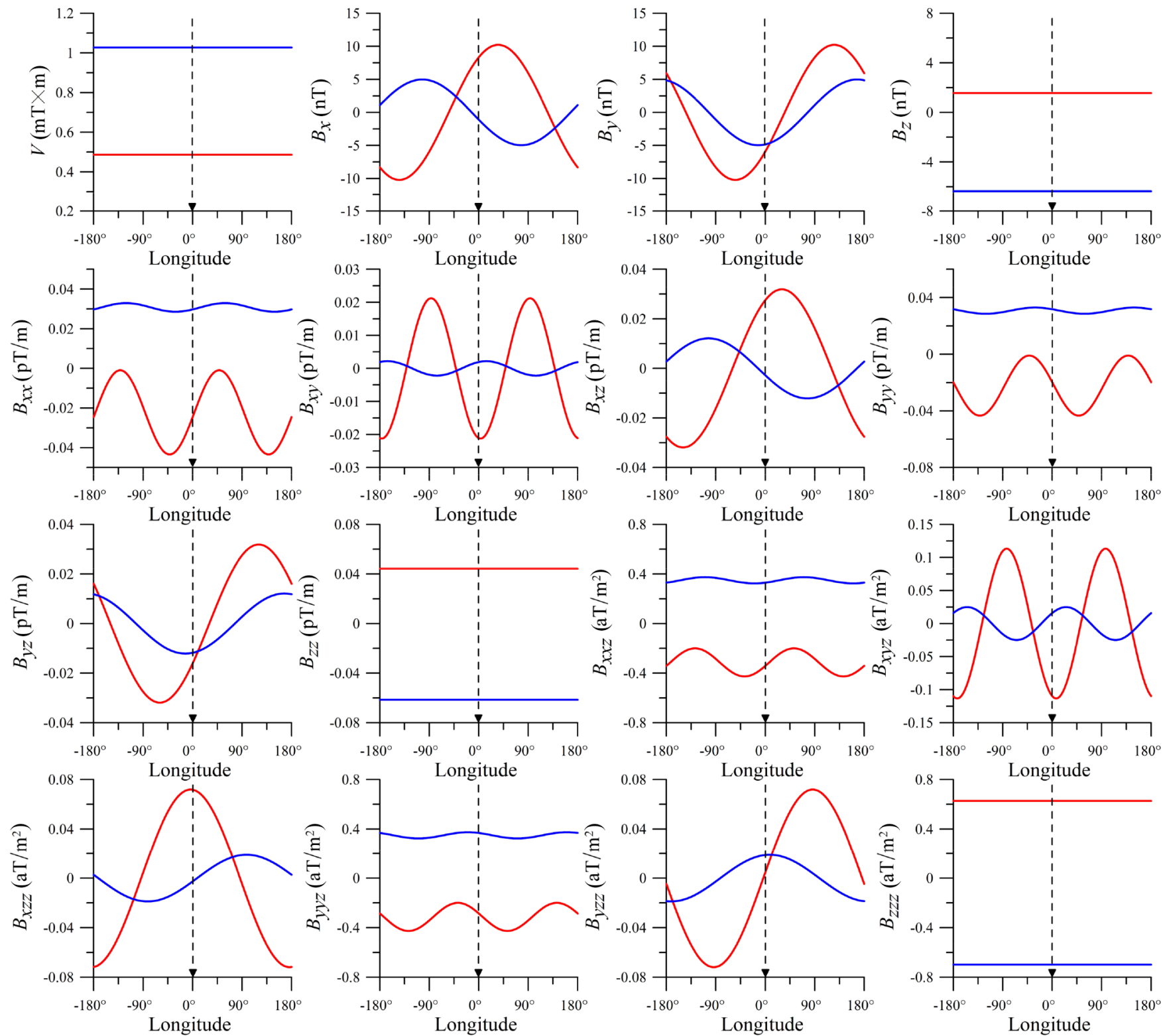

Figure 3. Limit values of the magnetic potential $(V)$, magnetic vector $\left(B_{x}, B_{y}\right.$ and $\left.B_{z}\right)$ and its gradients $\left(B_{x x}, B_{x y}, B_{x z}, B_{y y}, B_{y z}\right.$ and $\left.B_{z z}\right)$ and third-order partial derivatives of the magnetic potential field $\left(B_{x x z}, B_{x y z}, B_{x z z}, B_{y y z}, B_{y z z}\right.$ and $\left.B_{z z z}\right)$ at the poles when the local reference frames vary from different meridians (the direction of $x_{\mathrm{P}}$ axes changing from different meridians to the poles). Red and blue lines indicate the magnetic effects at the North Pole and at the South Pole, respectively. The reference frame is specially defined that the $x_{\mathrm{P}}$ axis points to the meridian of $180^{\circ} \mathrm{E}$ (or $180^{\circ} \mathrm{W}$ ) at the North Pole and $0 \mathrm{f} 0^{\circ}$ at the South Pole and the $y_{\mathrm{P}}$ axis points to the meridian of $90^{\circ} \mathrm{E}$ at both poles. The values at both poles shown by black dashed arrows are used to plot the maps in Figs. 1 and 2.

\section{Code availability}

Supplementary software implementation is performed with the programming language $\mathrm{C} / \mathrm{C}++$. The source code and input data presented in this paper can be obtained by contacting the corresponding author via email or download from the Supplement related to the online version of this article.

\section{The Supplement related to this article is available online at doi:10.5194/gmd-8-1979-2015-supplement.}

Acknowledgements. This study is supported by the International Cooperation Project in Science and Technology of China (no. 2010DFA24580), the Hubei Subsurface Multi-scale Imaging Key Laboratory (Institute of Geophysics \& Geomatics, China University 
of Geosciences, Wuhan) (grant no. SMIL-2015-06) and State Key Laboratory of Geodesy and Earth's Dynamics (Institute of Geodesy and Geophysics, CAS) (grant no. SKLGED2015-5-5-EZ). Jinsong $\mathrm{Du}$ is sponsored by the China Scholarship Council (CSC). We would like to thank Mehdi Eshagh and another anonymous reviewer for their constructive comments. All projected figures are drawn using the Generic Mapping Tools (GMT) (Wessel and Smith, 1991).

The article processing charges for this open-access publication were covered by a Research

Centre of the Helmholtz Association.

Edited by: L. Gross

\section{References}

Backus, G. E., Parker, R., and Constable, C.: Foundations of Geomagnetism, Cambridge University Press, Cambridge, 1996.

Bird, P.: An updated digital model of plate boundaries, Geochem. Geophys. Geosyst., 4, 1027, doi:10.1029/2001GC000252, 2003.

Blakely, R. G.: Potential Theory in Gravity and Magnetic Applications, Cambridge University Press, New York, 1995.

Blakely, R. J. and Simpson, R. W.: Approximating edges of source bodies from magnetic or gravity anomalies, Geophysics, 51, 1494-1498, 1986.

Eshagh, M.: Non-singular expressions for the vector and gradient tensor of gravitation in a geocentric spherical frame, Comput. Geosci., 34, 1762-1768, 2008.

Eshagh, M.: Alternative expressions for gravity gradients in local north-oriented frame and tensor spherical harmonics, Acta Geophys., 58, 215-243, 2009.

Finlay, C. C., Maus, S., Beggan, C. D., Bondar, T. N., Chambodut, A., Chernova, T. A., Chulliat, A., Golovkov, V. P., Hamilton, B., Hamoudi, M., Holme, R., Hulot, G., Kuang, W., Langlais, B., Lesur, V., Lowes, F. J., Lühr, H., Macmillan, S., Mandea, M., McLean, S., Manoj, C., Menvielle, M., Michaelis, I., Olsen, N., Rauberg, J., Rother, M., Sabaka, T. J., Tangborn, A., TøffnerClausen, L., Thébault, E., Thomson, A. W. P., Wardinski, I., Wei, Z., and Zvereva, T. I.: International Geomagnetic Reference Field: the eleventh generation, Geophys. J. Int., 183, 1216-1230, 2010.

Friis-Christensen, E., Lühr, H., and Hulot, G.: Swarm: A constellation to study the Earth's magnetic field, Earth Planet. Space, 58, 351-358, 2006.

Gauss, C. F.: Allgemeine Theorie des Erdmagnetismus, in: Resultate aus den Beobachtungen des magnetischen vereins im Jahre 1838, edited by: Gauss, C. F. and Weber, W., (Leipzig, 1839), 1-57, 1838.

Golynsky, A., Bell, R., Blankenship, D., Damaske, D., Ferraccioli, F., Finn, C., Golynsky, D., Ivanov, S., Jokat, W., Masolov, V., Riedel, S., von Frese, R., Young, D., and ADMAP Working Group: Air and shipborne magnetic surveys of the Antarctic into the 21st century, Tectonophysics, 585, 3-12, 2013.

Harrison, C. and Southam, J.: Magnetic field gradients and their uses in the study of the Earth's magnetic field, J. Geomagn. Geoelectr., 43, 485-599, 1991.

Holmes, S. A. and Featherstone, W. E.: A unified approach to the Clenshaw summation and the recursive computation of very high degree and order normalized associated Legendre functions, J. Geodynam., 76, 279-299, 2002a.

Holmes, S. A. and Featherstone, W. E.: SHORT NOTES: extending simplified high-degree synthesis methods to second latitudinal derivatives of geopotential, J. Geodynam., 76, 447-450, 2002b.

Hsu, S. K., Sibuet, J. C., and Shyu, C. T.: High-resolution detection of geologic boundaries from potential-field anomalies: An enhanced analytic signal technique, Geophysics, 61, 373-386, 1996.

Ilk, K. H.: Ein eitrag zur Dynamik ausgedehnter KörperGravitationswechselwirkung, Deutsche Geodätische Kommission. Reihe C, Heft Nr. 288, München, 1983.

Kotsiaros, S. and Olsen, N.: The geomagnetic field gradient tensor: Properties and parametrization in terms of spherical harmonics, Int. J. Geomath., 3, 297-314, 2012.

Kotsiaros, S. and Olsen, N.: End-to-End simulation study of a full magnetic gradiometry mission, Geophys. J. Int., 196, 100-110, 2014.

Kotsiaros, S., Finlay, C. C., and Olsen, N.: Use of along-track magnetic field differences in lithospheric field modelling, Geophys. J. Int., 200, 878-887, 2015.

Langel, R. A. and Hinze, W. J.: The Magnetic Field of the Earth's Lithosphere: The Satellite Perspective, Cambridge University Press, Cambridge, United Kingdom, 1998.

Langlais, B., Lesur, V., Purucker, M. E., Connerney, J. E. P., and Mandea, M.: Crustal Magnetic Fields of Terrestrial Planets, Space Sci. Rev., 152, 223-249, 2010.

Lesur, V., Rother, M., Vervelidou, F., Hamoudi, M., and Thébault, E.: Post-processing scheme for modelling the lithospheric magnetic field, Solid Earth, 4, 105-118, doi:10.5194/se-4-105-2013, 2013.

Maus, S.: An ellipsoidal harmonic representation of Earth's lithospheric magnetic field to degree and order 720, Geochem. Geophys. Geosyst., 11, Q06015, doi:10.1029/2010GC003026, 2010.

Maus, S., Yin, F., Lühr, H., Manoj, C., Rother, M., Rauberg, J., Michaelis, I., Stolle, C., and Müller, R. D.: Resolution of direction of oceanic magnetic lineations by the sixth-generation lithospheric magnetic field model from CHAMP satellite magnetic measurements, Geochem. Geophys. Geosyst., 9, Q07021, doi:10.1029/2008GC001949, 2008.

Olsen, N. and the Swarm End-to-End Consortium: Swarm-Endto-End mission performance simulator study, ESA contract No. 17263/02/NL/CB, DSRI Report 1/2004, Danish Space Research Institute, Copenhagen, 2004.

Olsen, N., Hulot, G., and Sabaka, T. J.: Sources of the Geomagnetic Field and the Modern Data That Enable Their Investigation, in: Handbook of Geomathematics, edited by: Freeden, W., Nashed, M. Z., and Sonar, T., Springer, Netherlands, 106-124, 2010.

Olsen, N., Lühr, H., Finlay, C. C., Sabaka, T. J., Michaelis, I., Rauberg, J., and Tøffner-Clausen, L.: The CHAOS-4 geomagnetic field model, Geophys. J. Int., 197: 815-827, 2014.

Olsen, N., Hulot, G., Lesur, V., Finlay, C. C., Beggan, C., Chulliat, A., Sabaka, T. J., Floberghagen, R., Friis-Christensen, E., Haagmans, R., Kotsiaros, S., Lühr, H, Tøffner-Clausen, L., and Vigneron, P.: The Swarm Initial Field Model for the 2014 geomagnetic field, Geophys. Res. Lett., 42, doi:10.1002/2014GL062659, 2015 . 
Pedersen, L. B. and Rasmussen, T. M.: The gradient tensor of potential field anomalies: Some implications on data collection and data processing of maps, Geophysics, 55, 1558-1566, 1990.

Petrovskaya, M. S. and Vershkov, A. N.: Non-singular expressions for the gravity gradients in the local north-oriented and orbital reference frames, J. Geodynam., 80, 117-127, 2006.

Purucker, M., Sabaka, T., Le, G., Slavin, J. A., Strangeway, R. J., and Busby, C.: Magnetic field gradients from the ST-5 constellation: Improving magnetic and thermal models of the lithosphere, Geophys. Res. Lett., 34, L24306, doi:10.1029/2007GL031739, 2007.

Purucker, M. and Whaler, K.: Crustal magnetism, in: Treatise on Geophysics, vol. 5, Geomagnetism, edited by: Kono, M., Elsevier, Amsterdam, 195-237, 2007.

Purucker, M. E.: Lithospheric studies using gradients from close encounters of Ørsted, CHAMP and SAC-C, Earth Planet. Space, 57, 1-7, 2005.

Ravat, D.: Interpretation of Mars southern highlands high amplitude magnetic field with total gradient and fractal source modeling: New insights into the magnetic mystery of Mars, Icarus, 214, 400-412, 2011.

Ravat, D., Wang, B., Wildermuth, E., and Taylor, P. T.: Gradients in the interpretation of satellite-altitude magnetic data: an example from central Africa, J. Geodynam., 33, 131-142, 2002.
Sabaka, T. J., Tøffner-Clausen, L., and Olsen, N.: Use of the Comprehensive Inversion method for Swarm satellite data analysis, Earth Planet. Space, 65, 1201-1222, 2013.

Sabaka, T. J., Olsen, N., Tyler, R. H., and Kuvshinov, A.: CM5, a pre-Swarm comprehensive magnetic field model derived from over 12 years of CHAMP, Ørsted, SAC-C and observatory data, Geophys. J. Int., 200, 1596-1626, 2015.

Schmidt, P. and Clark, D.: Advantages of measuring the magnetic gradient tensor, Preview, 85, 26-30, 2000.

Schmidt, P. and Clark, D.: The magnetic gradient tensor: its properties and uses in source characterization, The Leading Edge, 25, 75-78, 2006.

Taylor, P. T., Kis, K. I., and Wittmann, G.: Satellite-altitude horizontal magnetic gradient anomalies used to define the Kursk magnetic anomaly, J. Appl. Geophys., 109, 133-139, doi:10.1016/j/jappgeo.2014.07.018, 2014.

Thébault, E., Purucker, M., Whaler, K. A., Langlais, B., and Sabaka, T. J.: The Magnetic Field of Earth's Lithosphere, Space Sci. Rev., 155, 95-127, 2010.

Wessel, P. and Smith, W. H. F.: Free software helps map and display data, EOS Trans. AGU, 72, p. 441, 445-446, 1991. 